\title{
Degree of polarization in laser speckles from turbid media: Implications in tissue optics
}

Jun Li

Gang Yao

Lihong V. Wang

Texas A\&M University

Optical Imaging Laboratory

Biomedical Engineering Program

3120 TAMU, College Station, Texas 77843-3120

\begin{abstract}
The degree of polarization (DOP) of laser-speckle fields, where the speckles were generated by a polarized laser beam incident upon two kinds of samples: ground glass and wax, was investigated within a single coherence area as well as over multiple coherence areas. For the surface-scattering ground glass, the incident polarization state was preserved in the speckle field, and hence the DOP remained at unity regardless of the area of detection. For the volumescattering wax, the polarization states varied with positions in the field, and consequently the DOP depended on the area of detection: the DOP decreased with an increasing area of detection, and only when the area was much smaller than the coherence area would the DOP approach unity. A numerical simulation explained the experimental observation. These results are important for the understanding of polarization phenomena in turbid media such as biological tissue. (c) 2002 Society of Photo-Optical Instrumentation Engineers. [DOI: 10.1117/1.1483313]
\end{abstract}

Keywords: degree of polarization; laser speckle; turbid media; tissue optics.

Paper TP-01 received Apr. 29, 2001; revised manuscript received Mar. 20, 2002; accepted for publication Mar. 29, 2002.

\section{Introduction}

Optical polarization has recently become an active area of research in tissue optics. Polarization has been recognized as a unique contrast mechanism in biomedical optical imaging. ${ }^{1-4}$ Polarization techniques have been employed to reject ${ }^{5}$ or accept ${ }^{6}$ multiply scattered light from turbid media. There have been a number of publications on transmission of polarized light through a scattering medium. For example, Sankaran et al. ${ }^{7}$ experimentally studied the degree of polarization (DOP) of scattered light from biological tissues and tissue phantoms. Because a coherent-light source was generally used in these experiments, speckle patterns played significant roles in the polarization measurements.

The statistics of laser speckle patterns, including partially polarized speckle patterns, was well described in Goodman's chapter. ${ }^{8}$ In fact, partially polarized speckle patterns have been studied extensively in recent years. ${ }^{9-12}$ Fercher and Steeger ${ }^{9,10}$ determined the theoretical first-order statistics of Stokes parameters and later verified the theory with experiments. Brosseau ${ }^{12}$ studied the statistics of normalized Stokes parameters and discussed potential applications. Freund et al. ${ }^{13}$ proposed microstatistics to describe the polarization behavior of a single coherence area in a speckle field. The work was focused on deriving polarization correlation functions for extracting information about the direction of the incident polarization from the speckle pattern. Tarhan and Watson ${ }^{14}$ further investigated the microstatistics; they measured the intensity at many points in a speckle pattern for a given polarization angle of the incoming laser beam and obtained the probability density distributions for the parameters in the statistics. However, these two studies did not evaluate the DOP at those points in the speckle field, which is a key

Address all correspondence to Lihong Wang. Tel: 979-847-9040; Fax: 979-8454450; E-mail: Iwang@tamu.edu parameter for the understanding of polarized speckle fields. Elies et al., ${ }^{15}$ in a more recent investigation on speckle polarization, observed the speckle field produced by light reflected from a polished aluminum sample with a charge-coupled device (CCD) camera. Their results showed that depolarization among multiple speckle grains increased with sample inclination although each speckle grain remained polarized.

In this paper, we report on an investigation of polarization in a speckle field formed by coherent light being transmitted through a surface-scattering medium (a ground-glass plate) or a volume-scattering medium (a wax plate). The degree of polarization, as well as the degree of linear polarization (DOLP) and the degree of circular polarization (DOCP), were measured both within a single coherence area and over multiple coherence areas and were further modeled theoretically. Although it is widely acknowledged that multiple scattering events in volume-scattering media can depolarize polarized incident light and hence reduce the DOP, our study demonstrated that the measured DOP depended significantly on the conditions of observation.

\section{Experiments and Simulation}

The experimental setup is shown in Figure 1. A diode laser (SDL, TC40, $850 \mathrm{~nm}$ ) emitted a beam of $1.5 \mathrm{~mm}$ in full width at half maximum diameter and of $60 \mathrm{~m}$ in coherence length. After passing through an optical isolator and a half wave retardation plate, the beam was horizontally linearly polarized with a DOP of 0.99 and an intensity fluctuation of $\sim 1 \%$, where the isolator and the retardation plate were used to prevent back reflection into the laser and to fine tune the orientation of the polarization, respectively. The beam was incident upon the sample to produce a speckle field by the transmitted

1083-3668/2002/\$15.00 @ 2002 SPIE 


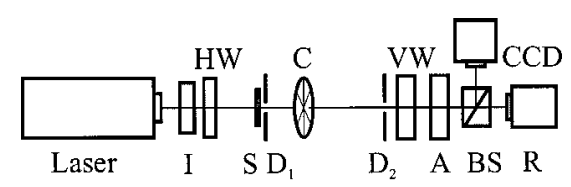

Fig. 1 Experimental setup. I, optical isolator; HW, half wave plate; $\mathrm{S}$, sample; $D_{1}$ and $D_{2}$, irises; $C$, chopper; $V W$, variable-wave plate; $A$, analyzer; $\mathrm{BS}$, nonpolarizing beam splitter; $\mathrm{R}$, photoreceiver.

light. An iris was set closely behind the sample to control the average size of coherence areas in the speckle field. Another iris was used to select a portion of the speckle field for observation. The selected light, after passing through a variablewave plate, a Glan-Thompson analyzer, and a nonpolarizing beam splitter, was detected by a large-area photoreceiver. The variable-wave plate was calibrated to an accuracy of $99 \%$ before measurements. A chopper operating at $900 \mathrm{~Hz}$ modulated the beam intensity, and the output of the photoreceiver was measured with a lock-in amplifier (Stanford Research Systems, SR510) to improve the signal-to-noise ratio. The chopper was set behind the first iris (close to the sample) to ensure that only the light emerging from the sample was modulated and detected. A CCD camera (Dalsa CA-D10256T, $256 \times 256$ pixels) was used to monitor the speckle pattern simultaneously. A 3-mm-thick wax plate was used as a volume-scattering sample, which multiply scattered the transmitted light. The wax sample was sufficiently thick to produce a speckle pattern of a high contrast, approaching the theoretical limit for unpolarized speckles $(1 / \sqrt{2})$. For comparison, a ground-glass plate was used as a surface-scattering sample, which scattered light only on the surface by deforming the phase front.

The average diameter $(d)$ of the coherence areas in the speckle field at the plane of detection, located at the second iris, was estimated by the following expression: ${ }^{16}$

$$
d=\frac{2.44 \lambda L}{D_{1}},
$$

where $L$ is the distance between the two irises, $D_{1}$ is the diameter of the first iris, and $\lambda$ is the optical wavelength. Equation (1) is the definition of the diameter of the Airy disk, which represents the minimum speckle size in a speckle pattern ${ }^{17}$ and can be used to estimate the average speckle size in a "fully developed" speckle pattern. By definition, a fully developed speckle pattern is completely polarized (DOP $=1)$. Although the speckle patterns in our experiments are not fully developed due to depolarization caused by multiple scattering, ${ }^{18}$ for simplicity, we took Eq. (1) as an approximation for the average speckle size in our study. For measurements of a single coherence area (multiple coherence area), $D_{1}$ was set to $0.1 \mathrm{~mm}(2 \mathrm{~mm})$, yielding an average diameter of coherence areas of $14.8 \mathrm{~mm}(0.74 \mathrm{~mm})$ at the detection plane with $L=711 \mathrm{~mm}$. By varying the area of detection determined by the size of the second iris $\left(D_{2}\right)$, one could select the number of detected coherence areas ranging between less than one and plurality, which was monitored with the CCD camera. The Stokes vector $\mathbf{S},{ }^{19}$ which describes a polarization state with four elements $\left(S_{0}, S_{1}, S_{2}\right.$, and $\left.S_{3}\right)$, was measured for each area of detection by adjusting the variable-wave plate and the analyzer

$$
\mathbf{S}=\left[\begin{array}{c}
S_{0} \\
S_{1} \\
S_{2} \\
S_{3}
\end{array}\right]=\left[\begin{array}{c}
I_{H}+I_{V} \\
I_{H}-I_{V} \\
I_{45^{\circ}}-I_{135^{\circ}} \\
I_{R}-I_{L}
\end{array}\right],
$$

where $I_{H}, I_{V}, I_{45^{\circ}}, I_{135^{\circ}}, I_{R}$, and $I_{L}$ are the light intensities measured with a horizontally linear analyzer, a vertically linear analyzer, a $45^{\circ}$ linear analyzer, a $135^{\circ}$ linear analyzer, a right circular analyzer, and a left circular analyzer, respectively. The DOP was then calculated by ${ }^{19}$

$$
\mathrm{DOP}=\left(S_{1}^{2}+S_{2}^{2}+S_{3}^{2}\right)^{1 / 2} / S_{0} .
$$

And, the DOLP and DOCP could be obtained by

$$
\begin{gathered}
\operatorname{DOLP}=\left(S_{1}^{2}+S_{2}^{2}\right)^{1 / 2} / S_{0}, \\
\mathrm{DOCP}=\left|S_{3}\right| / S_{0} .
\end{gathered}
$$

Figure 2(a) shows the DOP, DOLP, and DOCP measured within a single coherence area as functions of the size of the detection area. For the ground-glass sample, the DOP showed little variation associated with the size of the detection area and remained at $\sim 0.99$, which was approximately the same as that of the laser source. By contrast, for the wax sample, only the DOP of those small areas of detection was close to unity, and the DOP decreased as the area of detection was enlarged. Like the DOP, the DOLP and DOCP decreased with an increase in the area of detection for the wax sample. For the ground-glass sample, the DOLP and DOCP had nearly constant values: $\sim 0.99$ and $\sim 0$, respectively, which showed that linear-polarization states were maintained in the speckle field. Small fluctuations were seen in the DOCP measured from the ground-glass sample, which were due to low signal-to-noise ratios in the detection of the low-intensity circular-polarized component. Figure 2(b) shows the DOP, DOLP, and DOCP measured for multiple coherence areas. For both the groundglass and the wax samples, the trends in Figure 2(a) continued. It should be mentioned that the results in Figures 2(a) and 2(b) were not joined together because the measurements were not made under the same conditions as a result of the replacement of the first iris.

From the Stokes vectors obtained with the ground-glass sample, we found that the horizontally linear polarization state of the laser source was maintained in each measurement. In the measurements with the wax sample, a variation of the relative distribution of speckle intensity was observed with the CCD camera when the analyzer was rotated, indicating that the polarization states in the speckle field were nonuniformly distributed. Based on the effect of the scattering on light polarization, it is deduced that the multiple scattering events in the wax sample caused the distribution of polarization in the speckle field.

We investigated the probability density functions (PDFs) of Stokes parameters in the speckle field generated by the wax sample. Speckle patterns including multiple coherence areas were recorded with the CCD camera, which acted as a detector array. The Stokes parameters measured at each CCD pixel were taken for statistics. Figure 3 shows the probability density functions of the four Stokes parameters measured in the speckle field generated by the wax sample. The PDF of the 

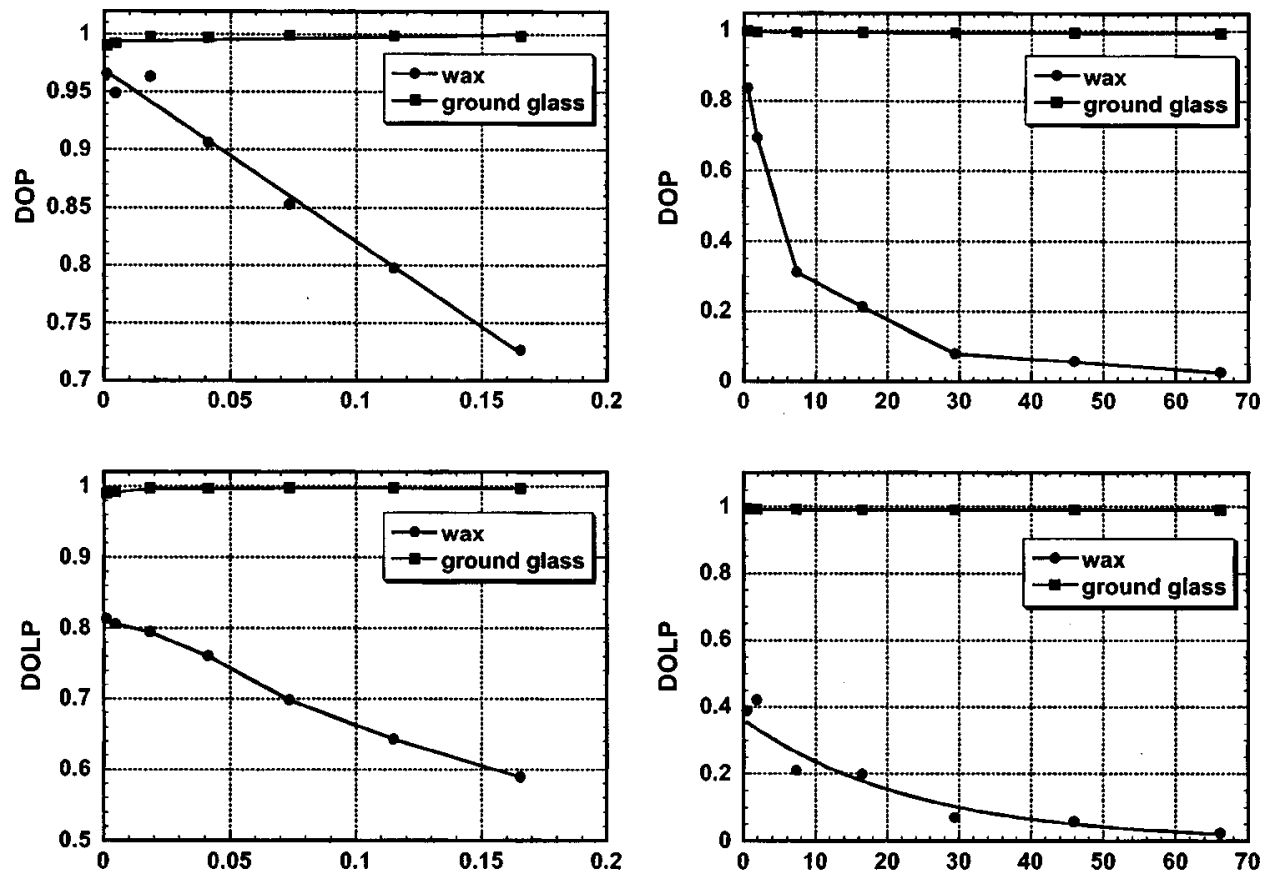

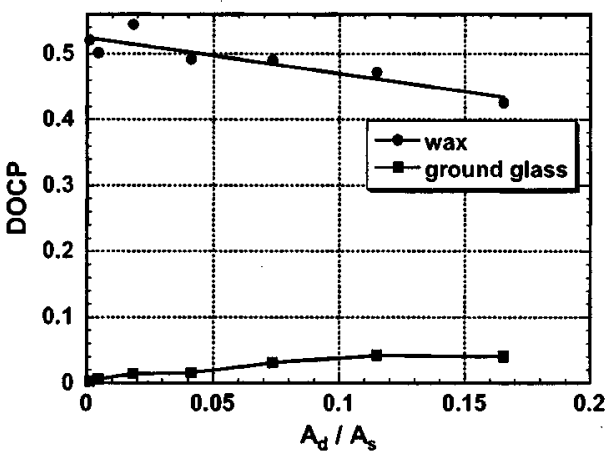

(a)

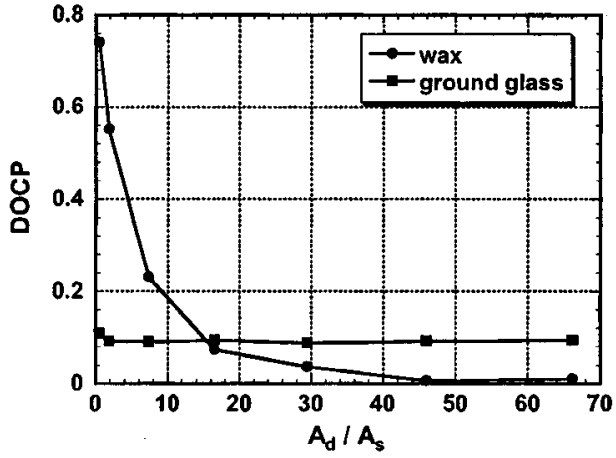

(b)

Fig. 2 Measured DOP, DOLP, and DOCP as functions of the area of detection. $A_{d}\left(=\pi d_{2}^{2} / 4\right)$ is the area of detection, where $d_{2}$ is the diameter of the second iris; $A_{s}\left(=\pi d_{s}^{2} / 4\right)$ is the average area of the coherence areas, where $d_{s}$ is the average diameter of the coherence areas. (a) Measurements within a single coherence area, where $A_{s}=171 \mathrm{~mm}^{2}$. (b) Measurements over multiple coherence areas, where $A_{s}=0.43 \mathrm{~mm}^{2}$.

first Stokes parameter, $S_{0}$, was similar to that obtained by Goodman $^{8}$ for the intensity of the sum of two speckle patterns, which was different from the negative exponential distribution of the fully polarized speckle pattern. The other three PDFs were symmetrically distributed. For comparison, a PDF of the first Stokes parameter of a speckle pattern generated by the ground-glass sample is given in Figure 3(a). It is seen that the distribution of this PDF is closer to the negative exponential distribution. Note that the DOPs corresponding to the two speckle fields in Figure $3(\mathrm{a})$ are $\sim 0.13$ and $\sim 0.99$, respectively. The variation of the PDF with the DOP agrees with Goodman's theory. ${ }^{8}$ According to the theory of Fercher and Steeger, ${ }^{9}$ in which the speckle field was described as a superposition of two fully developed uncorrelated linearly polarized speckle fields, the symmetrical distribution of the PDF of the second Stokes parameter indicates that the mean intensities of the two fields are the same.

Further, to well understand the phenomenon observed, we theoretically simulated the polarization states in speckle fields from a surface- and a volume-scattering medium, respectively. For the volume-scattering medium, both the polarization state and the phase of the transmitted optical field were assumed to be randomized by multiple scattering events. For the surface-scattering medium, only the phase of the transmitted optical field was assumed to be randomized as a result of the deformation of the phase front. In the simulation, the optical field at the first iris $\left(D_{1}\right)$ was represented by a Jones vector

$$
\mathbf{E}(\xi, \eta)=\left[\begin{array}{c}
E_{x}(\xi, \eta) \\
E_{y}(\xi, \eta)
\end{array}\right]=\left[\begin{array}{l}
E_{x_{0}}(\xi, \eta) e^{-j \phi_{x}(\xi, \eta)} \\
E_{y_{0}}(\xi, \eta) e^{-j \phi_{y}(\xi, \eta)}
\end{array}\right],
$$

where $E_{x}(\xi, \eta)$ and $E_{y}(\xi, \eta)$ are two orthogonal components of the field, and $(\xi, \eta)$ is the coordinate of a point in the plane where the first iris is located. A pupil function was applied to simulate the first iris, which gave the distribution of the opti- 

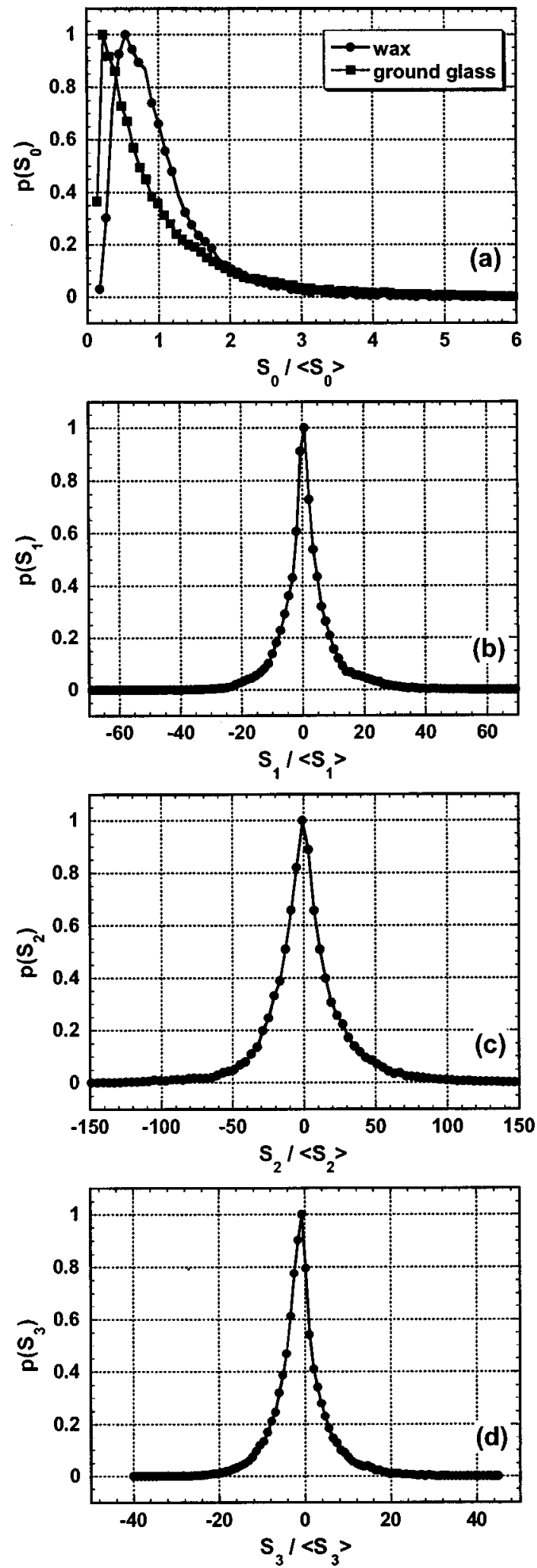

Fig. 3 Normalized probability density functions of Stokes parameters, which were measured in the speckle fields generated by the wax sample: (a) $S_{0}$, (b) $S_{1}$, (c) $S_{2}$, (d) $S_{3}$. The probability density function of the first Stokes parameter $S_{0}$ in the speckle field generated by the ground-glass sample is also given in (a) for comparison. $\left\langle S_{0}\right\rangle,\left\langle S_{1}\right\rangle$, $\left\langle S_{2}\right\rangle$, and $\left\langle S_{3}\right\rangle$ are the average values. cal field in the plane. For the surface-scattering medium, a horizontally linear polarization state with a constant $E_{x_{0}}$ and a zero $E_{y_{0}}$ was assumed, i.e.

$$
E(\xi, \eta)=\left[\begin{array}{c}
E_{x_{0}} e^{-j \phi_{x}(\xi, \eta)} \\
0
\end{array}\right]
$$

and the phase $\phi_{x}(\xi, \eta)$ was assumed to be randomized. For the volume-scattering medium, assumptions were made: $\arctan \left[E_{y_{0}}(\xi, \eta) / E_{x_{0}}(\xi, \eta)\right]$ was randomized between $-\pi$ and $\pi$, whereas the total optical intensity $\left[E_{x_{0}}^{2}(\xi, \eta)+E_{y_{0}}^{2}(\xi, \eta)\right]$ remained constant, and the phases $\phi_{x}(\xi, \eta)$ and $\phi_{y}(\xi, \eta)$ were randomized as well. For both of the media, the phase was evenly randomized between $-\pi$ and $\pi$. The two field components $E_{x}(\xi, \eta)$ and $E_{y}(\xi, \eta)$ were diffracted independently, which generated two independent speckle patterns in the far field. The diffraction processes were simulated by Fourier transforms

$$
\begin{aligned}
& E_{x}(x, y)=\mathrm{F}\left\{E_{x}(\xi, \eta)\right\}, \\
& E_{y}(x, y)=\mathrm{F}\left\{E_{y}(\xi, \eta)\right\},
\end{aligned}
$$

where $E_{x}(x, y)$ and $E_{y}(x, y)$ are optical fields at the point $(x, y)$ in the observation plane, and $\mathrm{F}\{\}$ denotes the Fourier transform. The final speckle pattern was generated by the summation of the two speckle patterns. The Stokes vectors of the speckle pattern were then calculated

$$
\left[\begin{array}{c}
S_{0} \\
S_{1} \\
S_{2} \\
S_{3}
\end{array}\right]=\left[\begin{array}{c}
E_{x}(x, y) E_{x}^{*}(x, y)+E_{y}(x, y) E_{y}^{*}(x, y) \\
E_{x}(x, y) E_{x}^{*}(x, y)-E_{y}(x, y) E_{y}^{*}(x, y) \\
E_{x}(x, y) E_{y}^{*}(x, y)+E_{y}(x, y) E_{x}^{*}(x, y) \\
j\left(E_{x}(x, y) E_{y}^{*}(x, y)-E_{y}(x, y) E_{x}^{*}(x, y)\right)
\end{array}\right] .
$$

The results of the simulation are shown in Figure 4. Figure 4(a) shows the variations of the DOP, DOLP, and DOCP with the size of detection area within a single coherence area. Figure 4(b) shows the results over multiple coherence areas. The simulation results agree with the experimental observation: a constant DOP of unity for the surface-scattering medium and a decreasing DOP for the volume-scattering medium as the area of detection increases. The DOLP and DOCP decrease with enlargement of the area of detection for the volumescattering medium, whereas they remain constant for the surface-scattering medium. Because of the statistical nature of a speckle field, the experimental results and the simulation results can be compared only qualitatively. Figure 5 displays the four Stokes-vector components of a segment of the speckle field from the volume-scattering medium corresponding to the maximum area of detection in Figure 4(a). It is clearly seen that the profiles are different among the four components. This agrees with the experimental observation from the wax sample and indicates that the Stokes vectors (polarization states) and the DOPs can vary from point to point in the speckle field, even within a single coherence area. This conclusion differs from the previous findings in speckle fields formed by light reflected from surface-scattering media. ${ }^{15}$ 

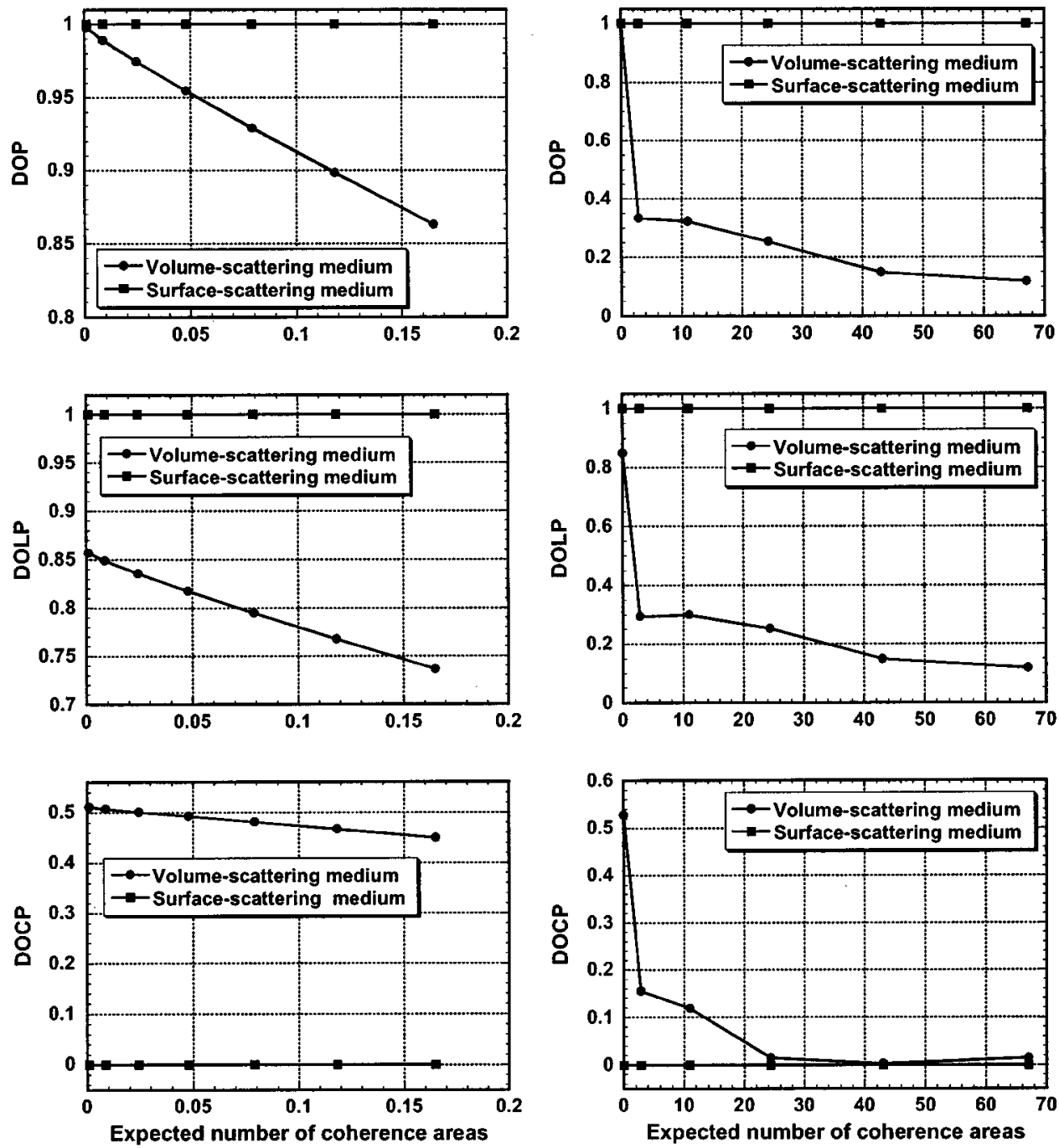

(a)

(b)

Fig. 4 Results of the simulation, where the expected number of coherence areas is defined as the area of detection divided by the expected area of the coherence areas. (a) The variations of DOP, DOLP, and DOCP within a single coherence area. (b) The variations of DOP, DOLP, and DOCP over multiple coherence areas.

\section{Discussion and Conclusions}

The results from our surface-scattering medium are obvious: because the speckle field is formed by the diffraction of an optical field with a single polarization state, the speckle field maintains the original polarization. For the volume-scattering medium, the independent diffraction processes of $E_{x}$ and $E_{y}$ create two orthogonal speckle fields polarized in the $x$ and $y$ directions, respectively. The vector sum of the two orthogonal speckle fields yields the total speckle field. Although the polarization states before diffraction are randomized, each point in the total speckle field has a DOP of unity because its resultant $E_{x}$ and $E_{y}$ components have a particular ratio of amplitude and a particular phase relation. Of course, the polarization states at different points in the total speckle field are statistically different from each other because both the ratio of amplitude and the phase between the two orthogonal speckle fields vary from point to point. The Stokes vector for an area including more than one such point is then determined by summing the Stokes vectors of all of the points in the area. As a result, the DOP of the area is less than unity and decreases statistically as the area is enlarged because more points are included in the enlarged area. It is worth noting that, because of their statistical nature, polarization states and DOPs can be different even for detection areas of the same size.

We conclude that the measured DOP, DOLP, and DOCP in a speckle field that is generated by a volume-scattering medium depend on the size of the detection area: they decrease with an increasing area of detection, and only the DOP of an area much smaller than a coherence area is close to unity. This conclusion is important for the understanding of polarization phenomena in tissue optics, where polarized coherent light is applied and a speckle field is generated. When the DOP, DOLP, and DOCP of a speckle field from a turbid medium such as biological tissue are measured, the above properties should be considered, especially if the measurement is made from a small area in the field. The fact that these parameters 

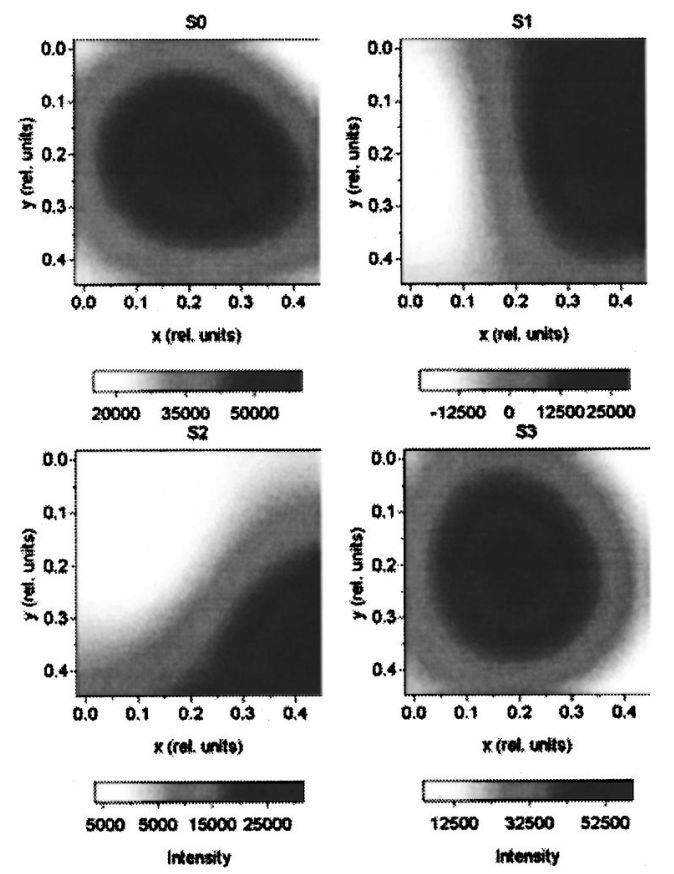

Fig. 5 The Stokes-vector components for a volume-scattering medium within a coherence area corresponding to the maximum in Figure 4(a).

may vary statistically even for areas of the same size should be taken into account as well. Moreover, if a speckle field was observed in reflection mode from a piece of biological tissue, contributions from both the rough surface and the multiply scattering light should be considered.

It is useful to compare our conclusions with the DOP in a heterodyne detection scheme such as the one used in optical coherence tomography (OCT). It was found that the DOP in OCT maintains a value of unity as long as the scattering sample is stable during data acquisition regardless of how many speckles are detected. ${ }^{20}$ OCT is an amplitude-based detection system that uses an interference heterodyne. OCT detects the electric field of only the coherent part of the backscattered light. The electric field of the light from various locations on the detector surface is projected onto the analyzing polarization state and then added in amplitude. Equivalently, the electric field vectors of the light from the various locations of the detector are summed, and the vector sum is then projected onto the analyzing polarization state. As a result of this coherent-detection scheme in OCT, a DOP of unity is maintained despite scattering.

\section{Acknowledgments}

We thank S. Jiao for experimental assistance. This project was sponsored in part by National Institutes of Health Grant No.
R01 CA71980; National Science Foundation Grant No. BES9734491; and Texas Higher Education Coordinating Board Grant No. 000512-0123-1999.

\section{References}

1. G. Yao and L.-H. Wang, "Two-dimensional depth-resolved Mueller matrix characterization of biological tissue by optical coherence tomography," Opt. Lett. 24, 537-539 (1999).

2. A. H. Hielscher, J. R. Mourant, and I. J. Bigio, "Influence of particle size and concentration on the diffuse backscattering of polarized light from tissue phantoms and biological cell suspensions," Appl. Opt. 36, 125-135 (1997).

3. J. F. de Boer, T. E. Milner, M. J. C. van Gemert, and J. S. Nelson, "Two-dimensional birefringence imaging in biological tissue by polarization-sensitive optical coherence tomography," Opt. Lett. 22, 934-936 (1997).

4. S. G. Demos and R. R. Alfano, "Optical polarization imaging," Appl. Opt. 36, 150-155 (1997).

5. V. Sankaran, K. Schonenberger, J. T. Walsh, Jr., and D. J. Maitland, "Polarization discrimination of coherently propagating light in turbid media," Appl. Opt. 38, 4252-4261 (1999).

6. S. L. Jacques, J. R. Roman, and K. Lee, "Imaging superficial tissues with polarized light," Lasers Surg. Med. 26, 119-129 (2000).

7. V. Sankaran, M. J. Everett, D. J. Maitland, and J. T. Walsh, Jr., "Comparison of polarized-light propagation in biological tissue and phantoms," Opt. Lett. 24, 1044-1046 (1999).

8. J. W. Goodman, "Statistics properties of laser speckle patterns," in Laser Speckle and Related Phenomenon, J. C. Dainty, Ed., pp. 9-75, Springer, Berlin (1975).

9. A. F. Fercher and P. F. Steeger, "First-order statistics of Stokes parameters in speckle fields," Opt. Acta 28, 443-448 (1981).

10. P. F. Steeger and A. F. Fercher, "Experimental investigation of the first-order statistics of Stokes parameters in speckle fields," Opt. Acta 29, 1395-1400 (1982).

11. R. Barakat, "The statistical properties of partially polarized light," Opt. Acta 32, 295-312 (1985).

12. C. Brosseau, "Statistics of the normalized Stokes parameters for a Gaussian stochastic plane wave field," Appl. Opt. 34, 4788-4793 (1995).

13. I. Freund, M. Kaveh, R. Berkovits, and M. Rosenbluh, "Universal polarization correlations and microstatistics of optical waves in random media," Phys. Rev. B 42, 2613-2616 (1990).

14. I. I. Tarhan and G. H. Watson, "Polarization microstatistics of laser speckle," Phys. Rev. A 45, 6013-6018 (1992).

15. P. Elies, B. LeJeune, F. LeRoyBrehonnet, J. Cariou, and J. Lotrian, "Experimental investigation of the speckle polarization for a polished aluminium sample," J. Phys. D 30, 29-39 (1997).

16. J. P. Mathieu, Optics, Pergamon, New York (1975).

17. J. C. Dainty, "Introduction," in Laser Speckle and Related Phenomenon, J. C. Dainty, Ed., pp. 1-7, Springer, Berlin (1975).

18. A. E. Ennos, "Speckle interferometry," in Laser Speckle and Related Phenomenon, J. C. Dainty, Ed., pp. 203-253, Springer, Berlin (1975).

19. R. A. Chipman, "Polarimetry," in Handbook of Optics, M. Bass, Ed., Optical Society of America, Washington (1995).

20. S. Jiao, G. Yao, and L.-H. V. Wang, "Depth-resolved twodimensional Stokes vectors of backscattered light and Mueller matrices of biological tissue measured by optical coherence tomography," Appl. Opt. 39, 6318-6324 (2000). 